\title{
Experiencia migratoria y vinculación internacional de investigadores uruguayos que deciden retornar al país
}

\author{
Migratory experience and international linkage of \\ Uruguayan researchers who decide to return to the \\ country
}

Experiência migratória e vinculação internacional de pesquisadores uruguaios que decidem retornar ao país

\section{Sofía Robaina ${ }^{1}$}

Programa de Población, Universidad de la República, Uruguay

sofia.robaina@cienciassociales.edu.uy 


\title{
Resumen
}

Este artículo se propone abordar dos aspectos de la movilidad de investigadores uruguayos: sus decisiones migratorias y los vínculos académicos que desarrollan internacionalmente. El objetivo es aportar tanto a la comprensión de los motivos asociados a la emigración y al retorno de investigadores uruguayos como al conocimiento acerca de los vínculos académicos transnacionales que estos desarrollan. Se realiza un abordaje cualitativo basado en la realización de entrevistas a investigadores que luego de una permanencia en el exterior deciden retornar al Uruguay. El análisis permite concluir que la movilidad y el intercambio internacional de investigadores uruguayos son promovidos por las restricciones del contexto nacional para formarse y dedicarse a la investigación; específicamente por las limitaciones comparativas del medio local en términos de oferta de posgrados, recursos materiales y humanos e infraestructura para hacer investigación.

Palabras clave: internacionalización de la ciencia; movilidad científica; vinculación internacional.

\begin{abstract}
This paper aims to deal with two aspects of the mobility of Uruguayan researchers: their migration decisions and the academic links they develop internationally. The objective is to contribute both to the understanding of the reasons associated with the emigration and the return of Uruguayan researchers, and to the knowledge about the transnational academic links they develop. A qualitative approach is carried out based on conducting interviews with researchers who, after a residence time abroad decide to return to Uruguay. The analysis allows us to conclude that the mobility and international exchange of Uruguayan researchers are promoted by the restrictions of the national context to be trained and to dedicate themselves to research; specifically due to the comparative limitations of the local scene in terms of postgraduate offer, infrastructure and, material and human resources to do investigation.
\end{abstract}

Keywords: Internationalization of science; scientific mobility; international linkage.

\section{Resumo}

Este artigo tem como objetivo abordar dois aspectos da mobilidade dos pesquisadores uruguaios: suas decisões de migração e as ligações acadêmicas que desenvolvem internacionalmente. O intuito é contribuir para a compreensão das razões associadas à emigração e ao retorno dos pesquisadores uruguaios e gerar conhecimento sobre os vínculos acadêmicos transnacionais que eles desenvolvem. Uma abordagem qualitativa é realizada com base na realização de entrevistas com pesquisadores que, após uma estadia no exterior, decidem retornar ao Uruguai. A análise permite concluir que a mobilidade e o intercâmbio internacional de pesquisadores uruguaios são promovidos pelas restrições do contexto nacional para se capacitar e dedicar à pesquisa, especificamente pelas limitações comparativas do ambiente local em termos da oferta de pós-graduação, insumos, recursos humanos e infraestrutura para pesquisa.

Palavras-chave: Internacionalização da ciência; mobilidade científica; ligação internacional. 


\section{Introducción}

Este artículo se propone abordar dos aspectos de la movilidad de investigadores uruguayos: sus decisiones migratorias y los vínculos académicos que desarrollan internacionalmente. La relevancia de estudiar este fenómeno se sustenta en, por lo menos, tres argumentos.

En primer lugar el investigador es una categoría de recurso humano calificado que está especialmente expuesto a la posibilidad de migrar: la migración científica es tan antigua como la ciencia, establecía Dedijer (1971). Como se desarrollará más adelante, la actividad científica y académica se caracteriza en forma creciente por su fuerte internacionalización, quiere decir que la propia carrera y dinámica de la actividad presiona a la movilidad internacional, siendo el intercambio académico internacional algo intrínseco a la propia tarea de investigar. Esa centralidad de la movilidad internacional en la actividad científica dota todo el proceso migratorio de los investigadores de características propias: desencadenantes, duración, vínculos, retorno, entre otras.

En segundo lugar, los investigadores, si bien son un subgrupo pequeño dentro de los recursos humanos calificados, son clave para impulsar procesos de desarrollo en toda economía de conocimiento; la disponibilidad y aprovechamiento de capacidades en ciencia, tecnología e innovación (CTI) es fundamental para el desarrollo económico y social de un país, por lo que contar con recursos humanos dedicados a la producción de conocimiento es crucial para el avance nacional. Si, como se establecía antes, el investigador está especialmente expuesto a la posibilidad de migrar, entonces es un fenómeno que requiere mayor atención. La emigración definitiva de investigadores podría significar la pérdida de recursos que son cruciales para la resolución de problemas locales que afectan el desarrollo, lo cual, considerando que el flujo se orienta fundamentalmente en dirección sur-norte, contribuiría a la profundización de las disparidades económicas y sociales al abrir aún más la brecha en términos tecnológicos y de conocimiento a escala mundial (Oteiza, 1969; 1998).

En tercer lugar, y a pesar de los dos puntos anteriores, las especificidades y motivos asociados a la movilidad de los investigadores han sido escasamente estudiadas.

Este artículo tiene por objetivo aportar a la comprensión de los motivos asociados a la emigración y al retorno de investigadores uruguayos $\mathrm{y}$ al conocimiento acerca de los vínculos académicos transnacionales que estos desarrollan. El análisis se basa en entrevistas realizadas a investigadores uruguayos que luego de un período en el exterior deciden volver al Uruguay, por lo que se abordan las motivaciones tanto detrás de la emigración como del retorno al país. Respecto a los vínculos académicos internacionales, se analizan aquellos que tienen lugar desde el exterior con Uruguay, como con la comunidad internacional una vez que se produce el retorno. Se indaga acerca de las modalidades en que se producen y en cuáles son sus principales promotores.

El artículo se organiza en cinco secciones. Luego de esta introducción, en la segunda sección se enmarcará conceptualmente la movilidad científica dentro del marco más general de la creciente internacionalización de la actividad académica y científica, y se referirá a los factores que la literatura indica como algunos de sus principales dinamizadores. En la tercera sección se presenta el abordaje metodológico empleado, y en la cuarta se presentan los resultados. La quinta sección reúne las principales conclusiones.

\section{Migración científica}

Diferentes trabajos han alertado sobre la importancia de evitar estudiar el fenómeno de la migración calificada como un todo homogéneo, desconociendo la variedad de situaciones y perfiles dentro de la misma, y sobre la relevancia de tomar en cuenta la naturaleza diversa de los recursos humanos calificados (Iredale, 2001; Ackers, 2005; Baruffaldi y Landoni, 2012). Abordar la migración calificada como un agregado 
homogéneo implica una simplificación analítica que enmascara la diversidad de talentos, sus motivaciones y características específicas (Solimano, 2005), porque la decisión de migrar y hacia dónde hacerlo no se toma en el vacío, sino que es tomada en forma racional en un marco específico de lógicas, posibilidades y constricciones.

El foco de este artículo se coloca en una categoría de recursos humanos calificados, en particular los investigadores, o en términos de Solimano (2005), los "talentos académicos". Se asume como definición de investigador la propuesta por la Organización para la Cooperación y Desarrollo Económico (OCDE) en el Manual de Frascati (OCDE, 2002) y tomada por la Red de Indicadores de Ciencia y Tecnología (RICYT), por lo que se considerará que "los investigadores son profesionales que se dedican a la concepción o creación de nuevos conocimientos, productos, procesos, métodos y sistemas, y también a la gestión de los proyectos respectivos" (OCDE, 2002, p. 99).

Lo anterior justifica la relevancia de explicitar y tener en cuenta el contexto y las características específicas de la actividad a la que se dedican. Específicamente, refiero a la fuerte internacionalización de la actividad científica, que si bien constituye un rasgo histórico del desarrollo de la ciencia y la tecnología, asociado a la dinámica de formación de recursos humanos, a los procesos de investigación y difusión del conocimiento (RICYT, 2007), se intensifica crecientemente en los últimos tiempos (Albornoz, Luchilo, Arber, Barrere y Raffo, 2002). Esto significa que las propias características de la carrera y la actividad científica presionan a la movilidad internacional; hay una expectativa de

2 Solimano (2005) propone diferenciar tres categorías de recursos humanos calificados: i) talentos vinculados directamente con la producción de bienes y servicios, ii) talentos académicos (científicos y estudiantes dedicados a la producción de conocimiento), y iii) talentos de sectores sociales y culturales (aquellos dedicados a la provisión de algún servicio social crítico como la salud, y aquellos que producen bienes culturales y desarrollan actividades creativas). movilidad en la carrera científica (Van Bouwel, 2010), que puede variar entre disciplinas y países pero que debe ser considerada a la hora de interpretar la migración de investigadores y científicos (Ackers, 2005). El intercambio académico internacional es por tanto en la mayoría de los casos algo intrínseco a la propia tarea de investigar, o el "alma" de la actividad científica (Paterlini, 2002, citado en Ackers, 2005), y esa centralidad dota a todo el proceso migratorio de los investigadores de características propias: desencadenantes, duración, vínculos, retorno, entre otras.

\subsection{Los factores dinamizadores de la internacionalización de la investigación}

La internacionalización de la actividad científica se expresa a través de ciertos rasgos que se posicionan como elementos intrínsecos de la producción de conocimiento en la actualidad (RICYT, 2007), a saber: el crecimiento de la producción científica y proyectos de investigación de coparticipación internacional, la publicación en coautoría internacional, y en la resultante movilidad y vinculación creciente de los recursos humanos dedicados a la actividad científica.

Son diversos los factores que promueven la internacionalización de la producción de conocimientos y pueden clasificarse en dos, los de carácter extracientífico y aquellos vinculados a la actividad científica propiamente.

En el primer tipo de factores se ubica la creciente facilidad y abaratamiento de los transportes y comunicaciones, la creación y fortalecimiento de procesos de integración entre los países y la internacionalización de la educación superior (López, 2015).

En el segundo tipo de factores que redundan en una creciente internacionalización de la investigación, se encuentran los vinculados a ciertos rasgos de la actividad científica, a saber: i) la especialización disciplinar y la imposibilidad de contar con masa crítica en todos los 
campos disciplinares; ii) los crecientes costos de llevar a cabo investigación en ciertas áreas de conocimiento y la imposibilidad de contar con recursos suficientes que fuerzan a una mayor colaboración; iii) nuevos criterios de evaluación y otorgamiento de fondos que premian la coparticipación de distintos países en proyectos de investigación y artículos en coautoría internacional que adquieren mayor visibilidad e impacto. Si bien los dos primeros aspectos son indicados en un trabajo desarrollado por Katz y Martin (1997) en torno a los motores de la colaboración científica en general, aquí, considerando la pequeñez de la comunidad académica uruguaya y la baja inversión en ciencia y tecnología, se considera que la creciente especialización disciplinar y los crecientes costos para hacer investigación son factores que en Uruguay promueven la colaboración internacional y, por ende, el proceso de internacionalización de la investigación.

En el Uruguay la internacionalización de la comunidad científica y una mayor cultura de intercambio con otros centros académicos experimentan un importante crecimiento como consecuencia de la restauración democrática a partir de 1985 (Buti, 2002). Durante el período dictatorial un importante número de investigadores se vieron obligados al exilio, y en el exterior continuaron desarrollando su actividad científica, generaron vínculos, y se integraron a redes. Gracias a la ayuda de diferentes programas de ayuda al retorno que surgen con la restauración de la democracia muchos de los investigadores retornan con más capacidades, nuevas dinámicas y nuevos contactos. Se constituyen así redes de relaciones internacionales que facilitan la movilidad: esos vínculos luego se extienden a los grupos locales y a los estudiantes, lo que termina redundando en una mayor internacionalización de la academia uruguaya.

Antes de pasar a los aspectos metodológicos conviene explicitar que este trabajo parte de la noción que la migración de los investigadores jóvenes o en formación es la que constituye el núcleo del problema de la emigración de personas dedicadas a la ciencia y la tecnología.
Por un lado, porque se parte de la premisa de que los investigadores maduros, ya formados o con cierta trayectoria se desarrollan en marcos de estabilidad ocupacional, están más arraigados a la comunidad académica local y muchas veces se encargan de la dirección de grupos, todos factores que reducen la propensión a emigrar. Además, si como lo propone Van Bouwel (2010) se asume la movilidad internacional de investigadores como una inversión en su formación, de acuerdo con la teoría del capital humano a medida que tienen más edad los individuos estarán menos dispuestos a realizar dicha inversión, debido a que el horizonte para que esta tenga su retorno es más reducido. Por otra parte, la emigración que forma parte del ciclo de formación de una persona constituye en ocasiones el primer antecedente de una migración definitiva (Albornoz et al., 2002), o el primer eslabón de la cadena migratoria, por lo que merece especial atención.

\section{Abordaje metodológico}

Como se advierte en Ackers (2005), capturar la naturaleza y el impacto del fenómeno de la migración altamente calificada y el esfuerzo por comprender los procesos de decisión de la migración de científicos exige un abordaje capaz de ir más allá del análisis cuantitativo.

Son necesarios estudios cualitativos profundos, que permitan identificar los factores motivacionales que determinan la movilidad y cómo estos afectan las características de los flujos. Asimismo, en lo que al estudio de las vinculaciones académicas internacionales se refiere, el abordaje cualitativo tiene la ventaja de abordar vinculaciones y cooperaciones transnacionales que no se captan en la mayoría de los estudios sobre el tema. La mayor parte de los antecedentes que han analizado las colaboraciones científicas lo han hecho a través de las coautorías en publicaciones (García-Hernández, 2012; Jonkers y Cruz-Castro, 2013; Scellato, Franzoni y Stephan, 2015), por tanto se han basado en indicadores bibliométricos, lo que invisibiliza otras formas de colaboración. El abordaje aquí elegido permite analizar otras formas de colaboración e 
intercambio que van más allá de la coautoría, recuperando lo que los estudios bibliométricos denominan "colegas invisibles" (Scellato et al., 2015).

Para lograr comprender los motivos detrás de las decisiones migratorias -emigración y retorno- y los vínculos académicos transnacionales se efectuaron veinte entrevistas en profundidad a investigadores que habiendo residido en el exterior deciden retornar a Uruguay a través de un programa de ayuda al retorno de la Universidad de la República (UdelaR), el programa de "Contratación de científicos provenientes del exterior".

Los investigadores entrevistados residen al momento de la entrevista en Montevideo, se encuentran empleados en la Universidad de la República, y fueron seleccionados con base a un muestreo intencionado de acuerdo con dos variables de corte: el sexo y el área de conocimiento en que el investigador se desempeña. Se eligen tales dimensiones en el entendido que son dos dimensiones que, en tanto factores que inciden en la estratificación de la ciencia académica (Tomassini, 2012), condicionan las decisiones y trayectorias migratorias y académicas, así como las dinámicas usuales de producción de conocimiento. Además, se efectuó la selección de entrevistados en procura de la mayor cobertura también de regiones de procedencia y duración de la estadía en el exterior.

\section{Resultados}

\subsection{Las decisiones migratorias}

\subsubsection{Contexto y motivos asociados a la emigración}

Se hizo referencia antes a la fuerte internacionalización y expectativa de movilidad internacional que caracteriza la actividad científica, desde todos los tiempos y en forma creciente en la actualidad. Partiendo de la concepción de la ciencia como una institución con pautas y normas (formales e informales) que rigen el accionar de los actores (Glaser, 2001), entonces se podría esperar que los individuos dedicados a la actividad científica migraran internacionalmente porque así lo mandata la institución que integran, es decir, que en parte la agencia del individuo a la hora de manejar sus propias carreras y sus decisiones migratorias estuviera condicionada, si no restringida, por los mandatos y las normas impuestas por la actividad a la que se dedican.

Efectivamente, cuando se acude al testimonio de los propios investigadores es clara la influencia de las expectativas de la institución científica sobre sus decisiones académicas y de movilidad. Lo anterior se expresa a través de la naturalización del evento migratorio, al asumirlo como inevitable o como una decisión obvia en el marco de su trayectoria científica. Cuando se los consulta sobre los motivos de la emigración surgen respuestas como3:

\footnotetext{
"Yo tenía claro que me tenía que ir para afuera en algún momento porque un científico tiene que ver otras cosas, me parecía que por mi carrera era fundamental irme para afuera” (Sal_M_20).

"Era algo absolutamente natural. Lo supe desde que decidí hacer esta carrera" (Agr_M_13).
"[me voy] porque era obligatorio para la ca- rrera académica. Yo lo vi desde que era es- tudiante, los propios docentes de la carrera nos decían: 'Esto es un principio, pero luego ustedes tienen que hacer un posgrado en el exterior"' (Sal_V_4).

Sin embargo, la institución científica y sus mandatos no son los únicos factores que influencian el accionar de los individuos dedicados a la investigación y sus decisiones de movilidad; existen otros factores contextuales e individuales que también ejercen su influencia.

3 Siempre que se incluya una cita de las entrevistas será referenciada indicando el área de conocimiento del investigador: agraria, básica, salud, social y tecnológica (Agr, Bas, Sal, Soc, Tec), su sexo ( $\mathrm{V}$ o M), seguido de un número identificador de la entrevista (de 1 a 20). 
Si bien la predisposición a la movilidad es un denominador común, y es el telón de fondo de las decisiones migratorias de los investigadores entrevistados, el evento emigratorio se materializa en contextos familiares, personales y laborales específicos y por intermedio de elementos desencadenantes de naturaleza diversa.

Se desprende de las entrevistas que desde la perspectiva de los individuos existen diferentes contextos de vida que son más o menos propicios para la emigración.

En cuanto a la situación personal y familiar de los entrevistados antes de la partida predomina tanto la carencia de hijos como la soltería, y se percibe tal coyuntura familiar y estatus marital como facilitadores de la decisión de emigrar. Es reiterada la mención a la falta de ataduras al país de origen, lo cual se asocia con la libertad de tomar y vivir las decisiones individualmente:

\footnotetext{
"No tenía ni hijos, ni pareja, tenía total libertad... no tenía presión... la decisión fue bastante libre" (Sal_V_4).

"En aquel entonces no tenía familia, o sea que estaba solo, dependía solo de mí la decisión y me fui" (Sal_V_7).
}

La modelación de las decisiones migratorias por parte del curso de vida de los individuos ha sido verificada por diferentes estudios, tanto de los procesos migratorios en general (Gandini, 2014; 2015) como de la migraciones científicas en particular (González y Malpica, 2013).

Las entrevistas realizadas coinciden con la evidencia previa en cuanto a que la decisión de migrar se encuentra mediada por el particular itinerario de vida del individuo. A través de la forma en que los entrevistados narran y reflexionan acerca del contexto familiar de su decisión emigratoria, se evidencia el condicionamiento que los eventos vitales previos ejercen sobre la posibilidad de migrar. A su vez su testimonio acerca de la decisión emigratoria en el pasado está permeado por lo que es su realidad familiar actual -momento en que reflexionan sobre su emigración-, en que la totalidad de los entrevistados tiene pareja o hijos, lo cual condicionará (aunque de diferente manera a mujeres y varones) las decisiones migratorias subsiguientes.

A la oportunidad que significó el contexto vital y personal particular por el que se atravesaba al momento de emigrar, se suma el particular contexto laboral de los investigadores al momento en que se produce su emigración, que alimenta la percepción de inexistencia de ataduras o contribuye a un cálculo de costo de oportunidad favorable a la decisión emigratoria.

Se observa que la mayoría de los entrevistados o bien no tienen trabajo estable antes del evento emigratorio, o tienen uno que lo conciben como mal remunerado o de relativa inestabilidad. Por ejemplo, todos los casos que tuvieron empleo en la universidad previo a la emigración detentaban cargos de carácter interino o eran becarios, y se encontraban en su mayoría subempleados. La frustración derivada de su inserción laboral antes de la emigración es moneda corriente en el discurso de los entrevistados y actúa, aunque en ningún caso determinando, sí promoviendo la decisión emigratoria.

Entonces, la yuxtaposición de dos elementos favorece la decisión de emigrar: i) la predisposición migratoria alimentada por la fuerte internacionalización de la actividad científica, y ii) la percepción por parte de los investigadores de atravesar un contexto vital propicio o habilitante para concretar el proyecto emigratorio: $\mathrm{Ni}$ en lo personal ni en lo laboral existen ligaduras al país, muy por el contrario las particularidades de ambos ámbitos redundan en que se concibe la decisión emigratoria como una sin mayores costos.

A la predisposición emigratoria que resulta de todo lo anterior se agrega la acción de diferentes elementos que terminan por gatillar la decisión de salir del país. Hay una referencia a factores de carácter económico o político, que en coyunturas puntuales actuaron como promotores de la decisión emigratoria, pero la referencia mayoritaria es a factores de tipo académico como desencadenantes de la emigración. 


\subsubsection{Desencadenantes de naturaleza extracientífica}

Trabajos anteriores muestran que la movilidad científica se caracteriza en gran medida por una independencia de los factores económicos (Baruffaldi y Landoni, 2012); sin embargo, la evidencia que surge en el desarrollo de esta investigación indica que el advenimiento de la crisis socio-económica que asoló a Uruguay a inicios del año 2000, el surgimiento de ciertas dificultades e inestabilidad laboral, sumado en algunos casos a la adquisición de deudas, es lo que en varios casos termina por desencadenar la decisión de emigrar. La recesión económica nacional referida se refleja en un claro empuje emigratorio con una marcada profundización del saldo migratorio negativo (OIM, 2011), y como podemos deducir del relato de los entrevistados, los científicos no quedaron por fuera del alcance de dicha crisis. La misma redundó en tasas de desempleo sin precedentes que afectó a amplios fragmentos de la sociedad, y en lo que al ámbito científico se refiere, mermó significativamente el presupuesto universitario y el destinado a la investigación. Como para muchos uruguayos, también para los investigadores, seguramente muchos de ellos en formación, se concibió la emigración como una estrategia de salida de la crisis y una oportunidad de concretar expectativas de desarrollo profesional y laboral que no encontraban cabida en el medio local.

Otros aluden al contexto político como condicionante de la decisión emigratoria. En el marco de la dictadura militar, se intervino la Universidad de la República en 1973, lo que implicó el desalojo y la ocupación de todas sus instalaciones por parte de las fuerzas militares, se suspendieron las actividades docentes, se dispuso el arresto de los miembros del Consejo Directivo Central y la administración general de la Universidad de la República pasó a estar en manos del Ministerio de Educación y Cultura, todo lo cual significó el desmantelamiento de lo creado y avanzado en materia científica (Tomassini, 2012), y actuó en muchos casos impulsando la emigración del país. No es el caso de ninguno de los entrevistados en el marco de esta investigación el de la emigración forzosa por motivos políticos, pero sí se refieren a la situación política imperante en el país y su impacto en el medio universitario como promotor de su decisión de emigrar.

\subsubsection{Desencadenantes de naturaleza científica}

Entre los elementos propiamente desencadenantes de la decisión de emigrar predomina la alusión a factores estrictamente académicos, entre los que se destacan los vinculados a las carencias o desventajas propias del medio académico local. En ese sentido una de las razones más aludidas para emigrar es la inexistencia o pobreza de oferta de posgrados en el país, por lo que se concibe el proyecto emigratorio como recurso para el progreso académico.

En Uruguay la creación de ofertas de formación de posgrado experimenta un crecimiento importante a partir de la creación de la Comisión Académica de Posgrados (CAP-UdelaR) en el 2001, que apoya y promueve la creación institucional de cursos de especialización, maestrías y doctorados, pero hasta ese momento la estructura de posgrados era muy mínima, lo que generaba dependencia de la formación en el exterior, empujando a muchos a emigrar en busca de una especialización. La creciente demanda de formación de posgrado -vinculada a las aspiraciones de superación profesional y académica, pero también como resultado de las pautas de evaluación individuales e institucionales imperantes que tornan cada vez más importante la titulación posgradual- se enfrenta a una todavía débil oferta de formación en maestría y doctorado, lo que se resuelve en ocasiones a través de la emigración. La asimetría de la oferta temática y geográfica de la formación de posgrado (Sebastián, 2003), pauta la dirección de los flujos migratorios que buscan capacitación. Algunas citas que dan cuenta de lo anterior son:

\footnotetext{
"Necesitaba seguir estudiando y acá en ese momento no había ni sueños, ni miras de posgrado" (Agr_M_13).
} 
"En aquel momento no había posgrados en agronomía, si uno quería hacer un posgrado debía hacerlo en el exterior" (Agr_M_16).

"No había acá. En esa época recién se empezaban a crear los posgrados en humanidades..." (Sal_V_4).

"Quería hacer algo dentro de la agronomía pero más numérico, y un posgrado era la oportunidad de combinar las dos cosas. Pero acá no había. Todavía no hay" (Soc_V_10).

“... La única posibilidad de formarte era irte al exterior” (Tec_M_18).

Las citas anteriores ejemplifican cómo la proyección académica y el deseo de especialización en ciertas disciplinas - pero de todas las áreas cognitivas- estaba, y en algunos casos aún está, condicionada al proyecto emigratorio. Como correlato de lo anterior, la totalidad de los entrevistados cursaron en el exterior diferentes programas de posgrado (maestría, doctorado y posdoctorado).

Finalmente, también entre los factores académicos desencadenantes de la emigración se ubica la referencia a desventajas propias de la dinámica o realidad académica local. Especialmente la referencia en este caso se centra en los tiempos que conllevan los procesos de formación en el medio local, los sueldos de los investigadores y la precariedad laboral (multiempleo y subempleo), la falta de comodidades o infraestructura para hacer investigación, entre otros. A continuación algunas citas representativas.

\footnotetext{
"El sistema acá era bastante frustrante. Todo muy problemático...” (Bas_V_3).

\begin{abstract}
"Yo veía que la gente acá estaba con 35 años y seguía haciendo el doctorado. Yo no, no pensaba seguir siendo becaria a los 35 años... y dije; 'ta, me voy ahora, si total en este país no voy a tener nada, voy a ganar dos mangos, voy a tardar 80 años en hacer la tesis, para eso me voy y hago el doctorado en tres años y medio y después veo" (Sal_M_20).
\end{abstract}

Se ve en lo anterior cómo un ambiente académico deprimido o menos desarrollado en términos de oportunidades y recursos opera expulsando a los jóvenes investigadores del medio local. La contracara es el magnetismo que ejercen los centros de investigación de primer nivel (Ackers, 2005): los investigadores buscan centros de excelencia académica, donde puedan acceder al último y mejor equipamiento, buscan recursos y mejores salarios para investigar, quieren un contexto donde el científico tiene prestigio (Martin-Rovet, 2003).

\subsubsection{La elección del destino}

La influencia que ejerce la fuerte internacionalización de la actividad científica y la importancia otorgada a la formación en el exterior se verifica incluso en la elección del destino de la emigración, en el sentido que este muchas veces no es priorizado ni elegido, sino que resulta de la postulación a diferentes universidades en el exterior y se define por azar, en función del lugar donde se obtuvo la aceptación:

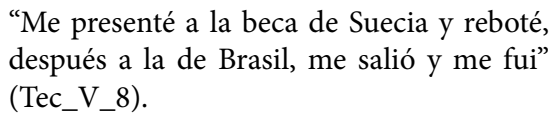

En otros tantos casos el destino de la emigración se definió por algún vínculo previo, directo o indirecto, con algún colega, grupo de investigación o departamento en el destino. Se observa aquí cómo las redes de relaciones internacionales preexistentes facilitan o constituyen el canal de la movilidad futura, y sustenta la idea trabajada en otros trabajos (Ackers, 2005) acerca de la importancia de las redes y contactos personales en la propensión a emigrar y en la elección del destino.

El tercer y último conjunto de motivos se vincula a factores familiares o de pareja. Coincide que son todas mujeres las que hacen alusión a este subconjunto de factores como el decisivo para resolver el destino de la emigración. O bien se define el destino por la opción o negociación con el marido, también académico; o bien se define en función de cuál es el destino más compatible con un proyecto de familia. La impronta del género se evidencia en todas las decisiones migratorias, tanto la de emigrar, como la de 
permanecer en el exterior, la de retornar al país de origen e incluso en la posibilidad de re-emigrar, lo que reivindica la consideración del género como variable relevante en los estudios de la migración calificada y científica específicamente, entre los que es frecuentemente invisibilizada.

\subsubsection{La decisión de retornar}

La decisión de volver al Uruguay se configura, al igual que la decisión emigratoria, por una combinación de factores de diferente tipo que operan a distinto nivel. Factores de carácter macro y meso se entretejen con las motivaciones individuales desencadenando la decisión de volver al país de origen (Lozano y Martínez, 2015), la cual se procurará desmenuzar y comprender, y en ningún caso se la concebirá como el final de la trayectoria migratoria.

La decisión del retorno no es fácil ni está libre de tensiones. Se identifica una asociación nítida entre la duración de la estadía en el exterior y la complejidad del proceso de decidir el retorno. Quienes permanecieron en el exterior por períodos más prolongados tuvieron la posibilidad de establecer vínculos académicos, humanos y laborales más sólidos en el país de acogida, en detrimento de los vínculos mantenidos con el Uruguay, lo que torna más difícil la decisión de volver al país. Diferente es la situación de quienes permanecen en el exterior por períodos más acotados, en proceso de formación, adquiriendo posiciones temporarias.

Como lo indican trabajos previos, el retorno puede verse motivado tanto por el incumplimiento como por el logro del objetivo que seguía la migración (Orrego y Martínez, 2015), y en ese sentido en algunos casos se observa una asociación entre los elementos primordiales en la decisión de emigrar y los que configuran la decisión de volver. Es el caso específico de aquellos que emigraron con el objetivo definido y puntual de realizar estudios de posgrado en el exterior y vuelven una vez que concretan su objetivo. Los investigadores a los que me refiero declaran que siempre existió la intención de volver a Uruguay y que nunca fue vivido como un proyecto definitivo, aunque en muchos casos terminan por tener una estadía en el exterior más extensa que la planificada originalmente.

Más allá de que muchos concibieron la vuelta al país desde el inicio del proyecto migratorio, el motivo de mayor mención en la concreción efectiva del retorno al Uruguay, que atraviesa el discurso de todos los entrevistados, es de tipo afectivo, y tiene que ver fundamentalmente con tres elementos: apego al país de origen, deseo de contribuir al desarrollo del país y factores familiares.

Emerge del testimonio de los entrevistados la existencia de una especie de efecto magnético que ejerce Uruguay sobre las decisiones migratorias. Una atracción que lejos de ser racional se funda en argumentos pura y exclusivamente afectivos, "volví porque soy uruguayo", "volví a las raíces", "por una cuestión de identidad con el país", "esta es tu tierra", son expresiones que claramente dan cuenta y razón de lo que se quiere transmitir. Se destaca especialmente la fortaleza de este argumento a la hora de hablar del retorno, y se evidencia que la identidad nacional no solo trasciende fronteras, sino que además perdura independientemente de la duración de la estadía en el exterior.

Aparece fuertemente también el retorno fundado en el deseo de contribuir al país. Esta idea se repite en varios entrevistados que conciben en Uruguay, por sus dimensiones, por las cercanías, la posibilidad de materializar su deseo altruista de hacer algún tipo de contribución al país:

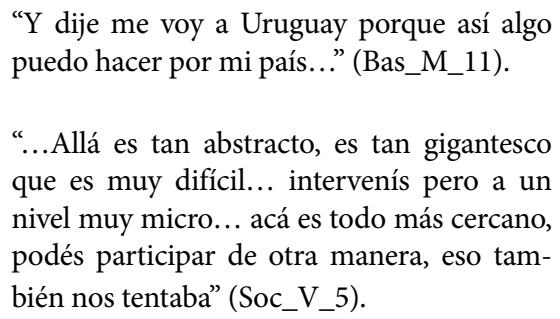

Finalmente, entre los elementos afectivos que impelen al retorno se encuentran los factores relacionados con la familia, específicamente el retorno se explica por tener familiares en el país o por desear iniciar el proyecto familiar en Uruguay. 
En este punto se justifica alzar la mirada sacando el foco del individuo retornante para colocarlo en la unidad familiar o conyugal, en la medida que el retorno se produce por una intersección entre la vida individual y la vida familiar, puntualmente a través de una priorización de la unidad familiar.

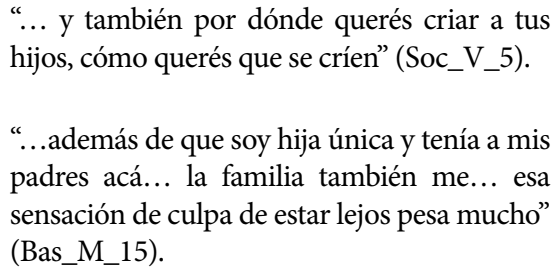

"Yo quería que mis hijas fueran uruguayas" (Soc_M_19).

La intención de establecerse para iniciar una familia en Uruguay es aludida por investigadoras mujeres y en todos los casos con relación a su edad:

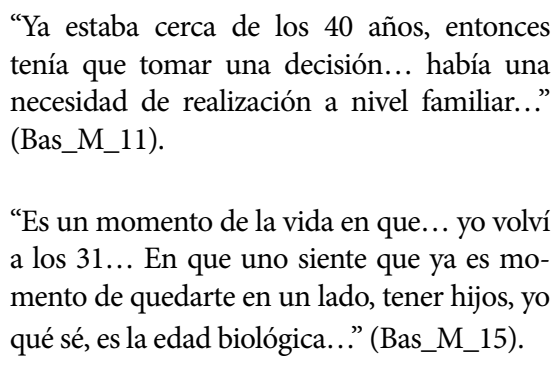
tenía que tomar una decisión... había una necesidad de realización a nivel familiar..." (Bas_M_11).

"Es un momento de la vida en que... yo volví a los $31 \ldots$ En que uno siente que ya es momento de quedarte en un lado, tener hijos, yo qué sé, es la edad biológica..." (Bas_M_15).

Como en la decisión de emigrar, se ve cómo el particular momento del curso de vida de las personas ejerce también su influencia sobre la decisión de volver. El no tener hijos era un contexto vital que se percibía como habilitante de la decisión emigratoria, ahora la decisión de tenerlos motiva la decisión migratoria inversa: volver al país. Se observa específicamente la fuerte influencia del modelo normativo predominante acerca del calendario en que debiera experimentarse el evento de la maternidad, internalizado fundamentalmente por las mujeres entrevistadas.

Aunque marginal, se identifica una referencia a elementos estrictamente académicos como influyentes en la decisión de volver a Uruguay, pero no que operaron desde el país atrayendo al investigador, sino con un efecto expulsor del ambiente de acogida en el exterior. El ambiente competitivo, dificultad de conseguir posiciones fijas y la consecuente inestabilidad laboral en el exterior actúan como impulsores del retorno. En un único caso la decisión es motivada por el antiguo jefe en Uruguay y justificada por un mayor desarrollo de la comunidad académica específica local.

\subsection{Vínculos académicos internacionales}

Se abordó la vinculación académica internacional que desarrollan los investigadores en dos momentos de su experiencia migratoria: se analizaron los vínculos con Uruguay durante la estadía en el exterior, y los vínculos con el exterior luego del retorno.

\subsubsection{Vínculos con el país de origen}

La vinculación con Uruguay desde el exterior se restringe a los de tipo personal y afectivo. La vinculación académica con el país es prácticamente nula durante la mayor parte de su estadía foránea, siendo esta una característica que atraviesa las experiencias de todos los investigadores entrevistados. Sin embargo, los vínculos académicos con el país son reactivados una vez que se decide el retorno, como forma de preparación del mismo.

De acuerdo con el planteo de Cassarino (2004; 2013) la preparación del retorno constituye una etapa crucial para que el retornado tenga una buena reincorporación y pueda eventualmente constituirse en agente de transformación y desarrollo. El análisis de las entrevistas habilita a afirmar que los investigadores que han emigrado se caracterizan por altos niveles de preparación del retorno. Son diversas las estrategias desplegadas por los investigadores ${ }^{4}$, pero se destaca la movilización de recursos de naturaleza intangible, en el sentido de Cassarino (2004), es decir de contactos, relaciones, habilidades.

Específicamente, y en línea con el planteo de la teoría de las redes sociales aplicada al estudio

4 Otra de las estrategias más extendidas para preparar el retorno es la adecuación de la agenda de investigación a los intereses del país o la comunidad académica local. 
de la migración de retorno (Jáuregui y Recaño, 2014), la principal y más extendida estrategia a la hora de preparar el retorno es a través de los contactos académicos. La creación de vínculos, el estrechamiento de relaciones o redes en el medio local, acercarse para conocer los temas en que se está trabajando, visitas previas para dictar cursos o participar de conferencias son claves para reforzar los contactos o hacerse conocido en el medio como forma de preparar el retorno. Como establece Ackers (2005), esos vínculos tornan al individuo más consciente acerca de sus oportunidades y facilitan la búsqueda de información y apoyo una vez que se tomó la decisión de retornar.

No obstante el esfuerzo por preparar el retorno para una mejor inserción en el medio de origen advierte la perspectiva estructuralista (Cassarino, 2004) que hay aspectos situacionales que el retornado no podrá calibrar con antelación, por lo que nunca podrá ser totalmente consciente de sus posibilidades reales y nunca estará completamente preparado para volver a su país. En ese sentido, uno de los principales obstáculos al que se enfrentan los investigadores al volver al país es la mala recepción o las resistencias por parte de los colegas en el medio en que se inserta, uno de los tantos imponderables a la hora de decidir y preparar el retorno.

Coincide la evidencia anterior con lo que observa Cerase (1974) respecto a las resistencias que el país y los contextos de inserción en origen ofrecen al retornado que llega con nuevas habilidades del exterior (return of innovation, según su tipología). Las relaciones de poder e intereses establecidos se resisten a la irrupción de nuevos actores con nueva formación, nuevas iniciativas y propuestas que puedan alterar el status quo y las estructuras vigentes. Se trata de un ejemplo de la argumentación de la perspectiva estructuralista del retorno al advertir sobre la relevancia de tomar en consideración la realidad y relaciones de poder e intereses locales a la hora de abordar el retorno y las posibilidades del retornado en el país de origen (Cassarino, 2004).
Los vínculos académicos con Uruguay no son sistemáticos y solo se activan con el cometido de preparar el retorno. Si bien proveen al futuro retornado de información sobre el medio de inserción futura, no lo previenen de resistencias y rigideces del medio difíciles de pronosticar desde la distancia.

\subsubsection{Vínculos académicos luego del retorno}

Pero los vínculos académicos internacionales sí se tornan activos una vez que el investigador vuelve al país. Los investigadores encuentran que la concreción de vínculos y colaboraciones con colegas del exterior favorece el acceso a capacidades inexistentes en el ámbito local, lo que actúa potenciando los procesos de producción de conocimiento.

La expansión de los contactos más allá de las fronteras nacionales constituye una estrategia paliativa del menor acceso a recursos en Uruguay. Fundamentalmente, son dos las falencias del medio local que los investigadores buscan resolver mediante la búsqueda de contactos y colaboración internacional: la menor masa crítica $^{5}$ y disponibilidad de capacidades en los campos específicos de conocimiento, así como el menor acceso a recursos materiales (exclusivamente a insumos y equipamiento) para la investigación.

La vinculación académica internacional que tiene lugar en mayor medida luego del retorno al país que antes del evento emigratorio, se produce fundamentalmente con el país de acogida, es decir que constituyen una continuación de las vinculaciones gestadas durante la estadía en el exterior.

Son dos los factores que se identifican como condicionantes o al menos facilitadores del

5 Un elemento adicional relacionado con la disponibilidad de masa crítica nacional, que actúa potenciando la colaboración internacional es la creciente especialización disciplinar (López, 2015) que acentúa la dificultad de contar en el medio local con especialistas con los que interactuar en todas las disciplinas y subdisciplinas. 
mantenimiento de las vinculaciones académicas con el exterior una vez que se retorna al país de origen: los encuentros presenciales esporádicos y la afinidad personal. Lo anterior es coincidente con lo que se evidenció en un trabajo sobre investigadores uruguayos quince años atrás (Buti, 2002), lo que nos permite concluir que -en el caso de las vinculaciones científicas- más allá del avance y las mejoras en las comunicaciones, el desplazamiento y el encuentro cara a cara no han podido ser sustituidos completamente por el uso de las nuevas tecnologías.

Las principales modalidades o vías a través de las que se concretan las colaboraciones y contactos en el exterior son cuatro: la invitación recíproca a conferencias o dictado de cursos, el envío de estudiantes de posgrado, participación en proyectos de investigación conjunta y las publicaciones en coautoría.

El envío de estudiantes al exterior se destaca especialmente y es prácticamente unánime la alusión a este medio de vinculación: para la realización de pasantías o estancias de investigación por parte de los estudiantes o para efectuar las fases experimentales de ciertos proyectos de investigación. A continuación algunos testimonios al respecto:

\footnotetext{
"Si vos entrás a mirar, buena parte de las cosas que tenemos es porque las hacemos con gente de afuera. Resultados y trabajo 100\% uruguayo no tenemos. Siempre a algún uruguayo conseguimos mandarlo a algún laboratorio a otro lado. Es decir, tenemos una idea, nos comunicamos con fulanito de tal lado que trabaja con esto y lo otro, va alguien allá, hace parte de ese trabajo allá, y cuando vuelve sacamos un artículo conjunto" (Sal_V_7).

“...En el proyecto $i+d$ que tengo, los responsables somos mi ex jefa de allá y yo, planeamos que el año que viene una estudiante se vaya para allá a hacer una pasantía larga por el proyecto y que haga los experimentos" (Sal_M_20).

“... A ellos les viene bien además porque en España es muy difícil conseguir estudiantes, entonces a ellos les aporta bastante porque
}

en definitiva tienen un estudiante trabajando casi gratis" (Tec_V_9).

Como se mencionaba antes, el destino de los estudiantes que se envían al exterior está en todos los casos determinado por el lugar de acogida del investigador durante su experiencia emigratoria, lo que da cuenta una vez más de cómo la experiencia en el exterior de un investigador puede motivar o reforzar la movilidad de los miembros de su grupo, investigadores o estudiantes más cercanos en el medio local, determinando a su vez el destino de tal movilidad y heredando estos los contactos y vínculos de aquel: una vez que la red de conocimiento se crea, provee el canal para la movilidad futura (Mahroum, 2001).

El envío de estudiantes es fundamental para garantizar una participación más activa del grupo local en el proyecto o trabajo de colaboración con el exterior, porque "si no existe la posibilidad de enviar a un estudiante o alguien que vaya al laboratorio en el exterior, entonces uno solo termina aportando la idea, y es otro el que la lleva a cabo" (Sal_V_7).

Se puede leer en la reflexión del investigador recién citado el temor a lo que se alerta en el Manual de Santiago (RICYT, 2007) como una amenaza de "una nueva forma de colonización" a través de la utilización de las capacidades de investigación de otros países y la obtención asimétrica de beneficios producto de la colaboración. No obstante el envío de estudiantes al exterior permite que los grupos locales accedan a capacidades, equipamiento, materiales e instalaciones que en Uruguay no son accesibles, lo cual es imprescindible y muy valorado por parte de los investigadores de las áreas naturales y exactas, ciencias de la salud y tecnológicas, varios trabajos lo han catalogado como una vinculación "asimétrica" (De Filippo, Barrere y Gómez, 2010), en el sentido que si bien en términos generales la colaboración internacional -en este caso el envío de estudiantes- suele aportar beneficios, estos no se distribuyen de la misma manera entre los países colaboradores. 
Por supuesto, la formación de estudiantes en laboratorios o universidades del exterior para la adquisición de nuevas técnicas, conocimientos y credenciales y el uso de equipamientos de otros países, redundan en otras formas de vinculación científica internacional. Ejemplos de lo anterior son la participación en proyectos de investigación conjuntos con colegas del exterior y las coautorías de publicaciones; modalidades de colaboración que también se ven estimuladas por los criterios universales de evaluación vigentes. El acceso a fondos internacionales frecuentemente exige la participación de varios países en proyectos conjuntos de investigación, e incluso los artículos publicados en coautoría internacional alcanzan mayor visibilidad, citación e impacto (De Filippo, Sanz y Gómez, 2007). En ese sentido la cita que sigue resulta muy elocuente:

Es poner cinco nombres para que te den un financiamiento. El extranjero se entera el día que te tiene que mandar la firma y el día que sacás un paper. Y si no lo incluís te rechazan. Ese tipo de tonterías las hacemos (Agr_V_6).

Esta cita problematiza y pone en cuestión la copublicación internacional como indicador válido de la colaboración internacional. En ese sentido, otros trabajos nos alertan y recomiendan precaución a la hora de diferenciar colaboración sin coautoría y copublicación en ausencia de una verdadera colaboración (López, 2015), lo que pone en cuestión la validez de los indicadores bibliométricos como medida de la colaboración científica internacional.

\section{Conclusiones}

Se abordaron aquí dos aspectos de la internacionalización de la actividad científica: los motivos asociados a la migración y las vinculaciones internacionales. Surge del análisis que para el caso uruguayo están los dos promovidos fundamentalmente por las características propias del medio académico y científico local.

Por un lado, se concluye que la decisión de emigrar se ve motivada en gran medida por una débil oferta nacional de posgrados que hace que las posibilidades de proyección académica y de especialización dependan del proyecto emigratorio. La formación doctoral y posdoctoral es fundamental para la consolidación de las capacidades en investigación y de la comunidad académica local. Entonces, la oferta asimétrica -en términos temáticos y geográficos- (Sebastián, 2003) y la todavía incipiente propuesta de programas de formación doctoral en el país tornan la movilidad internacional en un factor clave en el proceso de acumulación de capacidades en investigación.

Se observa asimismo la atracción que ejercen centros científicos y académicos de excelencia en el extranjero que, tanto por su oferta de formación como por su superioridad en términos de disponibilidad de equipamientos y condiciones para investigar, atraen a científicos uruguayos motivando su decisión de emigrar.

Por otro lado, también las vinculaciones académicas internacionales tienen como principal objetivo compensar falencias locales en lo que tiene que ver tanto con la disponibilidad de materiales, equipamiento e infraestructura, como de recursos humanos especializados. La principal modalidad de colaboración internacional es a través del envío de estudiantes al exterior, los que podrán gozar en el lugar de acogida de los medios inexistentes en el entorno uruguayo.

Se concluye de lo anterior que la internacionalización de la actividad científica en el caso uruguayo se ve especialmente alimentada por las diferentes condiciones para formarse y dedicarse a la investigación en uno y otro contexto, específicamente por las limitaciones comparativas del medio local en términos de oferta de posgrados, recursos e infraestructura para hacer investigación y escasa masa crítica.

Pero tales diferencias entre los contextos para hacer investigación no desaparecen y aún así el investigador toma la decisión de volver al Uruguay. Lo que cambia es la valoración que 
individuo efectúa respecto de los factores que ejercen influencia de un lado y del otro, la cual a su vez está mediada por el ciclo vital, familiar y profesional del sujeto migrante. Es así que arraigo e identidad nacional, motivos familiares y personales se destacan entre los motivantes del retorno al Uruguay.

El núcleo de la discusión en torno al vínculo entre migración de investigadores y desarrollo del país de origen se ubica en las posibilidades de transferencia de conocimiento adquirido en el exterior por parte de los investigadores migrantes al país y al medio académico de origen. De acuerdo con la literatura sobre el tema, tal transferencia y aporte de conocimiento y habilidades adquiridas pueden ocurrir en diferentes etapas del proceso migratorio de los investigadores: mientras se encuentra todavía en el exterior, y una vez que se produce el retorno.

Más allá de las especificidades propias de la transferencia de conocimiento en ambos momentos y si esta efectivamente tiene lugar o no -aspecto que aquí no fue analizado-, se puede concluir que la posibilidad de que ocurra depende en gran medida de motivaciones y estrategias individuales, en las que el ámbito científico-tecnológico local tiene mínima injerencia. Mientras la emigración está motivada por carencias del medio local y promovida por la atracción que ejercen centros de excelencia internacional, el retorno queda librado a motivaciones individuales y en general personales o afectivas. Asimismo, la experiencia de los investigadores entrevistados indica que la vinculación con Uruguay durante la estadía en el exterior consiste fundamentalmente en vínculos de carácter personal, siendo mayoritaria la ruptura de lazos con la comunidad académica nacional, los que son reactivados con fines utilitarios, como parte de la estrategia de preparación del retorno. Así, la posibilidad de que transferencias de conocimiento al país tengan lugar resulta muy menguada y con ella la posibilidad de que los investigadores puedan desde el exterior constituirse en agentes de desarrollo de su país de origen.

\section{Referencias}

Ackers, L. (2005). Moving People and Knowledge: The Mobility of Scientists within the European Union. International Migration, 45(5), 99-131.

Albornoz, M., Luchilo, L., Arber, G., Barrere, J. y Raffo, R. (2002). El talento que se pierde. Aproximación al estudio de la emigración de profesionales, investigadores y tecnólogos argentinos. Recuperado de http:// www.centroredes.org.ar/files/documentos/Doc.Nro4.pdf

Baruffaldi, S. H. y Landoni, P. (2012). Return Mobility and Scientific Productivity of Researchers Working Abroad: The Role of Home Country Linkages. Research Policy, 41(9), 1655-1665.

Buti, A. (2002). Científicos uruguayos en países del Mercosur: movilidad, redes y patrones culturales. Anuario Antropología Social y Cultural En Uruguay 2002-2003, (3), 125-135.

Cassarino, J. P. (2004). Theorising Return Migration: A Revisited Conceptual Approach to Return Migrants. International Journal on Multicultural Societies, 6(2), 253-279.

Cassarino, J. P. (2013). Teorizando sobre a migração de retorno: uma abordagem conceitual revisitada sobre migrantes de retorno. REMHU: Revista Interdisciplinar Da Mobilidade Humana, 21(41), 21-54.

Cerase, F. (1974). Expectation and Reality: a Case of Study of Return Migration from United States to Southern Italy. International Migration Review, 8(2), 245-262. 
Dedijer, S. (1971). Primeras migraciones. En W. Adams (Ed.), El drenaje de talentos (pp. 35-60). Buenos Aires, Argentina: Paidós.

De Filippo, D., Barrere, R. y Gómez, I. (2010). Características e impacto de la producción científica en colaboración entre Argentina y España. Revista Iberoamericana de Ciencia Tecnología y Sociedad, 6(16), 179-200.

De Filippo, D., Sanz, E. y Gómez, I. (2007). Movilidad de investigadores y producción en coautoría para el estudio de la colaboración científica. Revista Iberoamericana de Ciencia, Tecnología y Sociedad, 3(8), 23-40.

Gandini, L. (2014). Migración y curso de vida: la relación entre el cruce de fronteras internacionales y el proceso hacia la adultez. En M. Mora y O. de Olivera (Ed.), Desafíos y Paradojas (pp. 105-146). Ciudad de México, México: Colegio de México.

Gandini, L. (2015). ¿Escapando a la crisis? Un estudio comparativo de trayectorias laborales de migrantes argentinos en la Ciudad de México y Madrid. Recuperado de http://www.crim.unam.mx/web/node/309

García-Hernández, A. (2012). Las redes de colaboración científica y su efecto en la productividad. Un análisis bibliométrico. Investigación bibliotecológica, 27(59), 159-175.

Glaser, J. (2001). Macrostructures, Careers and Knowledge Production: a Neoinstitutionalist Approach. International Journal of Technology Management, 22(7/8), 698-715.

Gonzalez, A. y Malpica, C. (2013). International Mobility of Spanish Men and Women Doctorate Holder. Sociología y Tecnociencia, 3(3), 31-54.

Iredale, R. (2001). The Migration of Professionals: Theories and Typologies. International Migration, 395), 7-26.

Jáuregui, J. A. y Recaño, J. (2014). Una aproximación a las definiciones, tipologías y marcos teóricos de la migración de retorno. Revista Bibliográfica de Geografía y Ciencias Sociales, 19(1084), 1-32.

Jonkers, K. y Cruz-Castro, L. (2013). Research Upon Return: The Effect of International Mobility on Scientific Ties, Production and Impact. Research Policy, 42(8), 1366-1377.

Katz, J. S. y Martin, B. R. (1997). What is Research Collaboration? Research Policy, 26(1), 1-18.

López, M. P. (2015). Aportes para pensar las dimensiones internacionales de la investigación en América Latina. Revista Iberoamericana de Ciencia, Tecnología y Sociedad, 10(30), 173-197.

Lozano, F. y Martínez, J. (2015). Las muchas caras del retorno en América Latina. En F. Lozano y J. Martinez (Ed.), Retorno en los procesos migratorios de América Latina. Serie investigaciones $n^{\circ} 16$ (pp. 13-23). Río de Janeiro, Brasil: ALAP

Mahroum, S. (2001). Europe and the Immigration of Highly Skilled Labour. International Migration, 395), $27-43$.

Martin-Rovet, D. (2003). Opportunities for Outstanding Young Scientists in Europe to Create an Independent Research Team. Recuperado de http://fgimello.free.fr/documents/opportunities.pdf 
OCDE -Organización para la Cooperación y el Desarrollo Económicos-. (2002). Manual de Frascati. Propuesta de norma práctica para encuestas de investigación y desarrollo experimental. Recuperado de http://www.idi.mineco.gob.es/stfls/MICINN/Investigacion/FICHEROS/ManuaFrascati-2002_sp.pdf

OIM -Organización Internacional para las Migraciones-. (2011). Perfil migratorio del Uruguay. Montevideo, Uruguay: OIM.

Orrego, C. y Martinez, J. (2015). Retorno en la migración: una mirada a sus múltiples facetas. En F. Lozano y J. Martinez (Ed.), Retorno en los procesos migratorios de América Latina. Serie investigaciones $n^{\circ} 16$ (pp. 25-53). Río de Janeiro, Brasil: ALAP.

Oteiza, E. (1969). Emigración de profesionales, técnicos y obreros calificados argentinos a los Estados Unidos. Análisis de sus fluctuaciones. Buenos Aires, Argentina: Instituto Torcuato di Tella.

Oteiza, E. (1998). Drenaje de cerebros. Marco Histórico y Conceptual. En J. Charum y J. B. Meyer (Ed.), El nuevo nomadismo científico. La perspectiva latinoamericana (pp. 1-12). Bogotá, Colombia: Escuela Superior de Administración Pública.

RICYT -Red de Indicadores de Ciencia y Tecnología-. (2007). Manual de indicadores de internacionalización de la ciencia y la tecnología. Manual de Santiago. Recuperado de http://www.ricyt.org/manuales/ doc_view/1-manual-de-santiago

Scellato, G., Franzoni, C. y Stephan, P. (2015). Migrant Scientists and International Networks. Research Policy, 44(1), 108-120.

Sebastián, J. (2003). Estrategias de cooperación universitaria para la formación de investigadores en Iberoamérica. Madrid, España: Organización de Estados Iberoamericanos para la Educación, la Ciencia y la Cultura (OEI).

Solimano, A. (Ed.). (2005). The International Mobility of Talent: Types, Causes and Development. New York, US: Oxford University Press.

Tomassini, C. (2012). Ciencia académica y género: trayectorias académicas de varones y mujeres en dos disciplinas del conocimiento dentro de la Universidad de la República, Uruguay (tesis de maestría). Universidad de la República, Montevideo, Uruguay.

Van Bouwel, L. A. C. (2010). International Mobility Patterns of Researchers and Their Determinants. Recuperado de https://www.eurashe.eu/library/modernising-phe/mobility/scientific/WG4\%20R\%20 International\%20mobility\%20of\%20researchers.pdf 